\title{
Quelle est la motivation des internes de la première promotion du DES de médecine d'urgence?
}

\author{
What Motivates Students from the First National Promotion of Residents of the New French \\ Diploma of Emergency Medicine?
}

\author{
D. Douillet $\cdot$ C. Ammirati $\cdot$ C. Amsallem $\cdot$ P. Hausfater $\cdot$ J. Fonsegrive $\cdot$ C. Annweiler $\cdot$ P.-M. Roy $\cdot$ T. Pelaccia
}

Reçu le 4 mars 2019; accepté le 9 mai 2019

(C) SFMU et Lavoisier SAS 2019

Résumé Objectif : La motivation en formation étant positivement corrélée à l'implication, à la persévérance et aux performances des apprenants, nous avons souhaité identifier le profil motivationnel des étudiants de la première promotion du diplôme d'études spécialisées (DES) en médecine d'urgence (MU), afin d'identifier des leviers motivationnels et d'actions pour les enseignants permettant d'améliorer la qualité de la formation.

Matériel et méthodes : Nous avons mené une étude observationnelle descriptive multicentrique auprès des internes de première année de DES. Un questionnaire autoadministré en ligne a été envoyé à tous les internes. Cinq composantes de la motivation étaient explorées : la motivation intrinsèque (MI), la motivation extrinsèque (ME), la perception de valeur à la tâche (PVT), la perception de contrôlabilité (PCO) et le sentiment d'efficacité personnel (SEP).

D. Douillet $(\varangle) \cdot$ P.-M. Roy

Département de médecine d'urgence,

centre hospitalo-universitaire d'Angers,

F-49100 Angers, France

e-mail : Delphine.Douillet@chu-angers.fr

D. Douillet · C. Annweiler · P.-M. Roy

Département de médecine, faculté de santé,

université d'Angers, F-49100 Angers, France

C. Ammirati · C. Amsallem

Samu 80 et Centre de pédagogie active multidisciplinaire par simulation (SimUSanté), centre hospitalier universitaire

d'Amiens,

F-80080 Amiens, France

C. Ammirati

Département de médecine, faculté de santé,

université d'Amiens, F-80000 Amiens, France

\section{P. Hausfater}

Service des urgences, groupe hospitalier Pitié-Salpêtrière,

Assistance publique-Hôpitaux de Paris,

F-75013 Paris, France
Résultats : Trois cent soixante-quinze internes de la promotion 2017-2018 ont répondu, soit un taux de participation de $81,5 \%$. La MI était nettement supérieure à la ME (5,7 versus $3,8)$. Parmi les autres composantes de la motivation, la PVT était la plus élevée $(5,9)$ devant la $\mathrm{PCO}(5,2)$ et le $\operatorname{SEP}(5,2)$. Il n'y avait pas de corrélation entre les différentes composantes de la motivation et le rang de classement à l'ECN (épreuves classantes nationales) ni selon le fait que la MU était ou non leur premier choix.

Conclusion : La première promotion d'internes de $\mathrm{MU}$ obtient globalement des scores élevés de motivation. Deux leviers, sur lesquels les enseignants peuvent essayer d'agir prioritairement, ont été identifiés afin d'améliorer la motivation des internes.

Sorbonne Universités,

GRC-14 BIOSFAST (AP-HP), France

J. Fonsegrive

Service de médecine d'urgence, Samu 72,

centre hospitalier du Mans,

F-72037 Le Mans France

C. Annweiler

Service de gériatrie,

centre hospitalo-universitaire d'Angers,

F-49100 Angers, France

Robarts Research Institute, Department of Medical Biophysics, Schulich School of Medicine and Dentistry, the University of Western Ontario, London, ON, Canada

T. Pelaccia

Faculté de médecine, centre de formation et de recherche en éducation aux sciences de la santé (CFRPS),

université de Strasbourg, F-67085 Strasbourg, France

Samu 67, hôpitaux universitaires de Strasbourg,

F-67000 Strasbourg, France 
Mots clés Évaluation · Motivation · Médecine d'urgence · Levier motivationnel $\cdot$ Formation

\begin{abstract}
Aims: Motivation is positively correlated with involvement, perseverance, learning strategies and performance; we wanted to identify the motivational profile of students in the first class of the Specialized Diploma in Emergency Medicine, in order to identify motivational levers and actions for teachers to improve the quality of training.

Procedure: We have conducted a descriptive, multicenter, observational study of first year interns at DES. An online self-administered questionnaire was sent to all interns. The five components of motivation were explored: intrinsic motivation, extrinsic motivation, perceived value-to-task, perception of controllability, and sense of self-efficacy.

Results: Three hundred and seventy-five residents of the 2017-2018 class responded, representing a participation rate of $81.5 \%$. Intrinsic motivation was significantly higher than extrinsic motivation (5.7 versus 3.8). Among the other components of motivation, the perceived value-to-task was the highest (5.9) before the perception of controllability (5.2) then the sense of self-efficacy (5.2). There was no correlation between the different components of motivation and the rank of ECN (national classifying end of study examination programme) ranking, or whether or not emergency medicine was their first choice.

Conclusion: The first promotion of emergency medicine interns was that the overall scores were high on motivation. Two levers were identified on which teachers can primarily try to act in order to improve the motivation of the interns.
\end{abstract}

Keywords Assessment - Motivation · Emergency medicine · Motivational lever $\cdot$ Training

\section{Introduction}

La réforme du troisième cycle a permis à la première promotion d'internes de spécialité de médecine d'urgence (MU) de voir le jour en 2017 dans les 29 centres hospitalouniversitaires de France. Cette spécialité, reconnue officiellement depuis le 13 novembre 2015 (date de la publication de l'arrêté entérinant la création du diplôme d'études spécialisées [DES] de MU), l'est déjà dans de nombreux pays européens [1]. En France, avant la réforme, les internes choisissaient le plus souvent un DES de médecine générale puis se dirigeaient vers un diplôme complémentaire (DESC) en MU [2].

La motivation a été conceptualisée à la fin du XIX ${ }^{\mathrm{e}}$ siècle. Elle est définie comme « le construit hypothétique utilisé afin de décrire les forces internes et/ou externes produisant le déclenchement, la direction, l'intensité et la persistance du comportement » [3]. De nombreuses études démontrent les liens forts entre la motivation et l'apprentissage [4-8]. Dans les études médicales, il a notamment été démontré que plus le niveau de motivation est élevé, meilleures seront les stratégies d'apprentissage mobilisées, l'implication dans les activités d'apprentissage, la persévérance dans les tâches d'apprentissage et les études, et le niveau de performance atteint $[9,10]$.

Ryan et Deci [3] considèrent qu'il existe trois besoins psychologiques fondamentaux chez l'être humain adulte : le besoin de compétence, le besoin d'appartenance sociale et le besoin d'autodétermination. Ce dernier est défini comme le besoin qu'a l'être humain adulte de percevoir qu'il est seul à l'origine de ses choix et de ses décisions. Il exerce une forte influence sur la motivation, dans la mesure où un niveau élevé d'autodétermination traduit un engagement libre du sujet dans une activité qu'il privilégie en raison de l'intérêt, de la satisfaction, voire du plaisir qu'elle procure [3]. Il s'agit d'une forme de motivation dite " intrinsèque » $(\mathrm{MI})$. Un étudiant ayant une MI pour la MU trouverait par exemple une satisfaction importante à apprendre des contenus disciplinaires en lien avec la MU. On oppose couramment la MI à la motivation extrinsèque (ME), qui est liée à un engagement contraint dans une activité par la recherche de récompenses ou par la volonté d'éviter des sanctions. Par exemple, une forme extrinsèque de motivation à choisir la MU pourrait être guidée par l'image valorisante que cette spécialité peut renvoyer dans la société par les séries télévisées ou pour le complément de salaire généré par l'existence d'une activité de garde. Holland [11], en 2016, déclarait que les étudiants dans les cursus supérieurs étaient plus motivés par l'obtention du diplôme que par la volonté d'apprendre, surtout dans les cursus médicaux avec des processus de sélection importants.

Puisque motivation, apprentissage et performance sont intimement liés, repérer les leviers motivationnels chez les internes du DES de MU pourrait permettre aux enseignants et aux institutions d'agir positivement sur la motivation des étudiants et, donc, leur implication et leur persévérance, dans le but de favoriser un meilleur apprentissage et de maximiser la réussite. Selon Pelaccia et Viau [9], trois leviers motivationnels permettent d'agir positivement sur la motivation des apprenants. Il s'agit de la perception de valeur à la tâche (PVT), du sentiment d'efficacité personnel (SEP) et de la perception de contrôlabilité (PCO).

Ce travail avait pour objectif principal de déterminer le profil motivationnel (intrinsèque ou extrinsèque) des étudiants du DES de MU et d'identifier les leviers motivationnels (PVT, SEP, PCO) sur lesquels les enseignants de MU devraient s'appuyer pour renforcer la motivation des étudiants. L'objectif secondaire était d'évaluer si le rang de classement ou le fait d'avoir positionné ou non la MU en premier choix avait un impact sur les différentes composantes de la motivation, afin de répondre à la question suivante : « les personnes les mieux classées étaient-elles également les plus motivées?». 


\section{Méthode}

Il s'agissait d'une étude observationnelle descriptive menée sur le plan national de septembre 2018 à décembre 2018. La population étudiée comportait l'ensemble des internes de la phase socle du DES de MU 2017 résidant en métropole ou dans les DOM-TOM, à l'exclusion de ceux ayant fait valoir leur droit au remords. Les étudiants étaient invités par courriel à répondre à un questionnaire autoadministré en ligne $\left(\right.$ Google Form $\left.^{\circledR}\right)$. En cas de non-réponse au bout de sept jours, deux relances par courriel étaient réalisées à intervalle de sept jours.

Le questionnaire était structuré en deux parties (Annexe A) :

- première partie : données générales concernant la promotion et la formation.

Cette partie du questionnaire visait à recueillir des données descriptives sur le répondant, la formation dont il avait bénéficié et sur son rang de classement. L'objectif était de comparer ce niveau de motivation pour les différentes composantes au rang de classement à l'ECN ;

- seconde partie : évaluation de la motivation.

La seconde partie du questionnaire visait à mesurer les différentes composantes de la motivation. Le niveau de motivation a été mesuré au moyen d'une version adaptée en français et validée du questionnaire « Motivated Strategies for Learning Questionnaire » (MSLQ) développé dans sa forme princeps par Pintrich et al. [12]. Ce questionnaire comporte 26 items permettant d'évaluer la MI, la ME, le SEP, la PVT et la PCO :

- la perception de compétence ou SEP traduit la perception qu'a l'apprenant de ses capacités à réussir ce qui lui est demandé. La question correspondante est : « Suis-je capable de réaliser ce que l'on me demande de faire ? » [13] ;

- la PVT correspond à l'appréciation qu'a une personne de l'utilité, de l'intérêt et de l'importance de la tâche à réaliser. La question correspondante est : « À quoi ce que vous me demandez de faire ou d'apprendre va-t-il me servir? » [13];

- la PCO désigne le degré d'autonomie et de contrôle que perçoit un apprenant dans sa formation. La question correspondante est : «Ai-je mon mot à dire sur ce que l'on me demande de faire ? » [13].

Les valeurs des différentes composantes de la motivation ont été obtenues en calculant la moyenne des réponses obtenues aux questions correspondantes. Une moyenne élevée correspond à un niveau élevé pour la composante motivationnelle explorée $[12,14]$. Pour chaque question, les internes devaient renseigner leur degré d'accord avec la pro- position sur une échelle de Likert graduée de 1 (pas vrai du tout pour moi) à 7 (tout à fait vrai pour moi) (Annexe A).

Une phase préliminaire de validation du questionnaire a été réalisée pour évaluer la faisabilité et l'acceptabilité auprès d'un échantillon de 13 internes (soit 2,8\% de la population d'étude). À l'issue de cette phase et au regard des commentaires libres communiqués par les répondants, nous avons supprimé une question, reformulé la première partie du questionnaire et réagencé l'ordre des questions.

La participation à l'étude était volontaire et anonyme. Le protocole ainsi que le questionnaire ont été approuvés par le comité d'éthique du CHU d'Angers le 23 mai 2018 (numéro 2018/52).

\section{Analyse statistique}

Après clôture du questionnaire, une analyse a été réalisée post hoc. Nous avons d'abord calculé le taux de réponse, puis les données épidémiologiques des internes ayant répondu ont été comparées aux données des nonrépondants, principalement concernant le sexe, l'âge et le rang de classement, afin d'évaluer la présence ou non d'un éventuel biais de sélection. Ces comparaisons ont été réalisées en utilisant le test $\mathrm{du}_{\mathrm{Chi}}{ }^{2}$ ou le test de la somme des rangs de Wilcoxon, en fonction du type de variable étudiée. Les valeurs des différentes composantes de la motivation ont été décrites par la moyenne et l'écart-type. Des estimations du coefficient de corrélation de Spearman ont été calculées pour évaluer l'association entre les différentes composantes de la motivation et, d'une part, les rangs de classement à l'ECN 2017 et, d'autre part, le fait d'avoir ou non fait de la MU son premier choix. Une différence était considérée comme significative si $p$ inférieur à 0,05 . Les analyses statistiques ont été réalisées via le logiciel $\mathrm{R}$ version 3.5.1 (Free Software Fondation, Boston).

\section{Résultats}

Sur les 460 internes interrogés, 375 ont répondu au questionnaire, soit un taux de participation de $81,5 \%$ au niveau de la promotion nationale. Les internes n'ayant pas complété entièrement le MSLQ ont été exclus $(n=15)$. Les données manquantes dans la première partie concernaient la ville d'origine, le classement à l'ECN et quelques données sur la formation reçue. Vingt-trois erreurs dans le rang de classement ECN ont été identifiées lors de la comparaison au fichier national. Une imputation par la moyenne pour les données quantitatives a été utilisée pour gérer ces données manquantes ou aberrantes. Chez les répondants, le sex-ratio était de 0,9 (200 femmes/375).

L'âge moyen était de 26 ans $( \pm 2,4)$. Le classement moyen à l'ECN était de 5265 (avec un minimum à 408 et un 
maximum à $8193 \pm 1$ 698). Les caractéristiques des nonrépondants étaient similaires concernant l'âge $(p=0,32)$, le sexe $(p=0,21)$ et le rang de classement $(p=0,45)$. La spécialité « MU » était le premier choix pour 278 internes, soit $74,1 \%$ des répondants. Les étudiants dont la MU n'était pas le premier choix auraient choisi principalement l'anesthésieréanimation ou la médecine intensive-réanimation. Trentequatre internes déclaraient avoir pour projet de faire de la MU avant de commencer leurs études de médecine $(n=34 / 375[9,1 \%])$. Trente-trois pour cent des internes déclaraient s'être décidés depuis moins d'un an (123/375). Le choix de la MU a été fait pour $54,5 \%$ des répondants après leur passage dans un service d'urgence ou un Samu pendant leur externat $(n=204 / 375)$. Concernant les formations reçues lors de la première année de DES MU, $70 \%$ des internes $(n=263 / 375)$ avaient bénéficié de séminaires interrégionaux et $97,3 \%$ avaient participé à des journées de formation organisées localement, avec une moyenne de $9 \pm 5,3$. Les internes avaient bénéficié de formations par la simulation environ trois fois dans l'année $\pm 1,9$. Seuls $51,2 \%$ des internes déclaraient avoir un tuteur désigné pour leurs quatre années de formation $(n=192 / 375)$. Sur une échelle de Likert s'échelonnant de 1 à 9 et évaluant la satisfaction des répondants vis-à-vis de leur formation reçue, celle-ci était en moyenne de $7 \pm 1,3$.
Les résultats obtenus concernant les différentes composantes de la motivation sont reportés dans le tableau 1. La MI était nettement supérieure à la $\mathrm{ME}(5,7$ vs 3,$8 ; p=0,04)$. Concernant les autres composantes de la motivation, la PVT était la plus élevée $(5,9)$, devant la PCO $(5,2)$ et le $\operatorname{SEP}(5,2)$.

Les corrélations entre les classements à l'ECN, le fait que la MU soit ou non le premier choix des internes et les différentes composantes de la motivation sont reportées dans le tableau 2 et sur la figure 1. Aucune corrélation ne s'est avérée significative. La PVT et le classement semblaient être les plus corrélés, sans que ce résultat ne soit statistiquement significatif (coefficient de corrélation : 0,$095 ; p=0,07$ ). La PCO semblait être plus corrélée avec le fait de choisir ou non la MU en premier, sans atteindre le seuil de significativité (coefficient de corrélation $=-0,146 ; p=0,06$ ).

\section{Discussion}

Les scores de motivation sont globalement élevés chez les internes de première année du DES de MU. La MI et la PVT sont les plus élevées. La ME, qui correspond au fait d'agir sous la contrainte, est la composante motivationnelle dont le niveau est le plus faible.

Tableau 1 Composantes de la motivation des internes vis-à-vis de leur formation en médecine d'urgence $(n=375)$

\begin{tabular}{|c|c|c|c|}
\hline & Nombre de questions & Composante $* \dagger$ & $\begin{array}{l}\text { Coefficient } \alpha \\
\text { de Cronbach du MSLQ }\end{array}$ \\
\hline Motivation intrinsèque & 4 & $5,7 \pm 1,2$ & 0,74 \\
\hline Motivation extrinsèque & 4 & $3,8 \pm 1,6$ & 0,69 \\
\hline Sentiment d'efficacité personnelle & 8 & $5,3 \pm 1,3$ & 0,91 \\
\hline Perception de valeur à la tâche & 6 & $5,9 \pm 1$ & 0,88 \\
\hline Perception d'autonomie et de contrôle & 4 & $5,2 \pm 1,4$ & 0,68 \\
\hline
\end{tabular}

Tableau 2 Corrélation entre les composantes de la motivation et le classement à l'ECN

\begin{tabular}{|lll|}
\hline Tableau 2 Corrélation entre les composantes de la motivation et le classement à l'ECN & \multicolumn{2}{c|}{ DES** premier choix } \\
\hline Variable & Rang ECN* & $0,109(0,16)$ \\
\hline Motivation intrinsèque & $0,019(0,71)$ & $0,006(0,94)$ \\
Motivation extrinsèque & $0,015(0,78)$ & $-0,146(0,06)$ \\
Perception d'autonomie et de contrôle & $-0,043(0,40)$ & $0,057(0,46)$ \\
Perception de valeur à la tâche & $0,095(0,07)$ & $0,019(0,81)$ \\
Sentiment d'efficacité personnelle & $0,001(0,98)$ & \\
\hline$*$ ECN : épreuves classantes nationales ; **DES : diplôme d'études spécialisées & \\
Les données sont exprimées en valeur du coefficient de corrélation (valeur de p) & \\
\hline
\end{tabular}



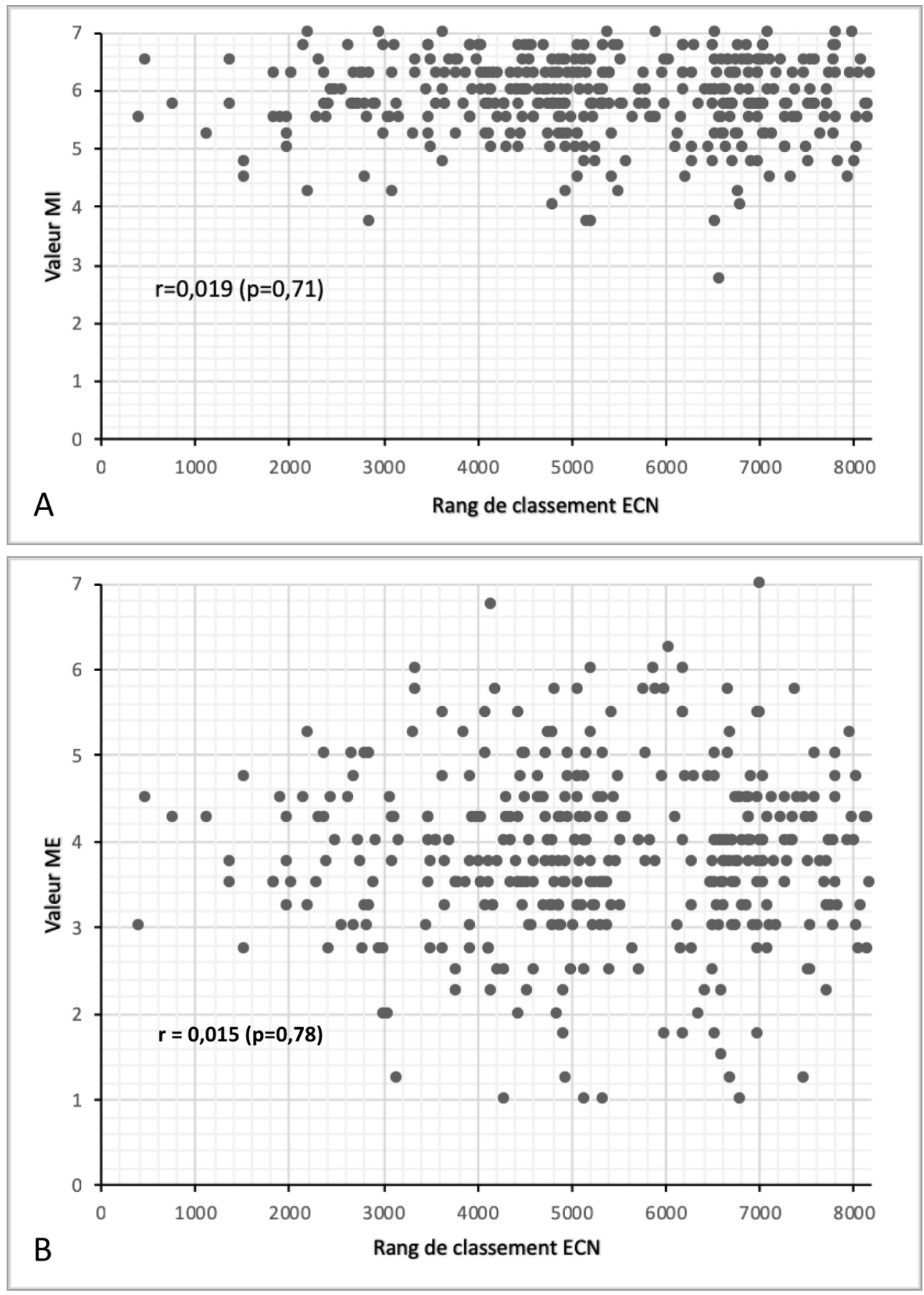

Fig. 1 A. Comparaison entre la motivation intrinsèque (MI) et le rang de classement à l'examen national classant (ECN). B. Comparaison entre la motivation extrinsèque $(\mathrm{ME})$ et le rang de classement à l'ECN

Le choix de la MU comme spécialité est rarement présent avant ou dès le début des études médicales $(9,1 \%$ de notre population). Un moment décisif dans le choix de la spécialité de MU est le passage en stage de MU (service d'accueil des urgences et/ou Samu). Un des facteurs explicatifs est sans doute l'importance du modèle de rôle lors de ces stages cliniques. Celui-ci est défini par Tiberius et al. comme «quelqu'un que les apprenants ont perçu comme digne d'être imité » [15]. Il a largement été mis en évidence que le fait d'observer des modèles de rôle experts et de s'inspirer de leurs actions et de leurs réflexions influence fortement le choix de carrière [16-18]. Au Royaume-Uni comme en France, le choix de carrière en MU se fait plus tard que pour d'autres spécialités. Selon Gallagher et al. [19], la réalisation d'un stage dans un service d'urgence où il existe un département universitaire en MU impliqué dès les premières 
années de la formation en médecine et/ou un accueil d'interne en MU majore la volonté des étudiants de choisir cette spécialité. La prise en charge des étudiants hospitaliers est donc primordiale afin de favoriser l'attractivité de la MU. En dehors de facteurs propres à l'étudiant (facteurs démographiques, traits de personnalité, valeurs morales), le choix de carrière se fait également en fonction de leur expérience clinique dans la spécialité ou de leur apprentissage en milieu académique, des représentations issues des discours sur la MU (par exemple, dans les médias ou de la part d'autres médecins), des perceptions, par les étudiants, des besoins de la société ainsi que de leur projection de leur vie professionnelle future [20-22]. Ces éléments constituent autant de facteurs d'attractivité de la spécialité de MU.

Un des résultats les plus marquants dans notre étude est la très nette supériorité de la MI $(5,7)$ par rapport à la $\mathrm{ME}(3,8)$. Ce résultat doit être interprété positivement, puisque comme nous l'avons souligné en introduction, plus le niveau de MI est élevé, meilleures sont les performances et la qualité des apprentissages [10,23]. Le faible niveau de ME diminue la sensibilité des étudiants à des incitations telles que les récompenses (par exemple, les bonnes notes) et les sanctions (par exemple, un redoublement). Il est en revanche nécessaire de maintenir un niveau élevé de MI. L'une des principales stratégies consiste à privilégier le recours à des méthodes actives d'enseignement, telles que la découverte ou la méthode des cas [8].

Les deux composantes de la motivation (qui constituent deux leviers motivationnels) dont les niveaux sont les plus bas sont la PCO et le SEP. Plusieurs stratégies ont été décrites dans la littérature scientifique afin d'agir efficacement sur ces deux composantes [8].

Le fait de permettre aux apprenants d'effectuer des choix en leur laissant des espaces de liberté dans la formation constitue la principale stratégie visant à agir positivement sur la PCO, dès lors que ce choix a un sens pour eux. Ainsi, laisser une flexibilité dans les lieux de stage, leurs ordres de réalisation, la possibilité de réaliser des DIU spécialisés complémentaires à leur formation pendant leur internat, l'accès à des formations spécialisées transversales sont des exemples de mesures pouvant permettre d'améliorer la perception de contrôle des étudiants. Il est par ailleurs important d'annoncer les objectifs d'évaluation et de faire en sorte que les résultats obtenus soient perçus par l'apprenant comme proportionnels aux efforts. Un échec chez un étudiant qui considère s'être beaucoup investi dans la préparation d'un examen diminue en effet sa PCO.

Le SEP est un déterminant complexe de la motivation, car il résulte de nombreuses interactions : les expériences passées, les expériences vicariantes (c'est-à-dire, le fait d'observer des pairs en situation de réussite ou d'échec), la persuasion verbale et les états émotionnels et physiologiques (l'anxiété peut diminuer le SEP) [9]. Afin d'agir positive- ment sur cette composante, il est d'abord important de renseigner régulièrement l'étudiant sur sa progression (par exemple, grâce à l'usage du portfolio), ce qui permet de mettre en valeur la place de l'évaluation formative. Il est ensuite souhaitable de fixer des objectifs pédagogiques de difficulté moyenne, car des objectifs trop complexes diminuent le SEP, et des objectifs trop simples à atteindre ne le modifient pas. Il est enfin crucial d'offrir à l'apprenant des rétroactions visant notamment à l'encourager lorsqu'il est performant et à le rassurer sur ses capacités lorsqu'il est en situation d'échec, en prenant notamment le soin de valoriser les éléments positifs de sa prestation, même en cas d'échec global par rapport à la tâche demandée. Ces différentes recommandations permettent de valoriser l'importance du tutorat, dont seulement un interne sur deux avait bénéficié dans notre étude.

Selon Bandura, les étudiants ayant un plus haut niveau de SEP obtiennent de meilleurs résultats [24]. Il est de même des étudiants dont le niveau d'autodétermination est le plus élevé [8]. L'absence de résultat similaire dans notre étude pourrait être expliquée par le fait que la variable utilisée (le rang de classement à l'ECN) est un mauvais indicateur de performance.

Nous souhaiterions enfin souligner que le profil motivationnel n'est pas différent selon que les étudiants aient ou non choisi la MU en premier. Il n'y aurait donc pas lieu de différencier, sur le plan motivationnel, des groupes d'étudiants sur la base de ce critère.

Notre étude est associée à un taux très élevé de participation, ce qui favorise la généralisation des résultats. L'hypothèse selon laquelle les étudiants les plus motivés sont également ceux qui ont le plus répondu est peu probable au regard $\mathrm{du}$ fait que les caractéristiques des répondants et des nonrépondants étaient similaires. L'existence d'un biais de sélection est donc peu vraisemblable. Cette étude est la première à s'intéresser à la motivation des étudiants en MU. Pour la mesurer, nous avons eu recours à des outils standardisés, associés à des niveaux élevés de validité et de fidélité [12].

Cependant, cette étude a été menée à la fin de la phase socle. Elle ne permet donc pas d'évaluer la motivation initiale des internes lors de leur entrée dans le troisième cycle. De nombreux facteurs, liés notamment aux stages, sont susceptibles d'avoir modifié la motivation des répondants entre le début et la fin de la première année. Il serait intéressant de répéter cette évaluation à la fin du DES pour déterminer l'évolution des composantes de la motivation (en particulier la MI et la ME). Une autre limite est le possible biais d'information sur ce recueil déclaratif. En effet, le questionnaire administré était relativement long (temps estimé à 15 minutes), et nous avons observé dans quelques cas $(<20)$ une cotation similaire sur les trois à quatre dernières questions. Les qualités psychométriques et métrologiques du questionnaire dans sa version française n'ont par ailleurs pas été évaluées. Afin de mieux connaître les facteurs qui expliquent la 
motivation des étudiants, il serait intéressant de compléter ce travail par la mise en œuvre d'entretiens semi-dirigés.

\section{Conclusion}

La première promotion d'internes de MU obtient globalement des scores élevés de motivation. Le SEP et la PCO sont les deux leviers grâce auxquels les enseignants peuvent espérer agir positivement sur la motivation. Une nouvelle analyse sera proposée en fin de phase de consolidation afin de déterminer l'évolution des composantes de la motivation dans cette population.

Liens d'intérêts : les auteurs déclarent ne pas avoir de liens d'intérêts.

\section{Annexe A. Questionnaire utilisé}

\begin{tabular}{|c|c|c|c|c|c|c|c|c|}
\hline \multirow[t]{2}{*}{$\begin{array}{l}\text { Composante } \\
\text { explorée }\end{array}$} & \multirow[t]{2}{*}{ Quelle est votre motivation dans le choix de cette formation spécialisée en médecine d'urgence? } & 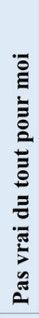 & 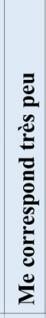 & 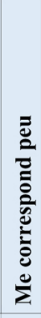 & 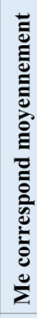 & 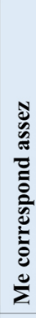 & 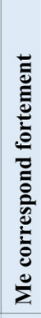 & 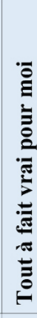 \\
\hline & & 1 & 2 & 3 & 4 & 5 & 6 & 7 \\
\hline MI & 1. Je préfère des sessions de formation qui me mettent au défi pour que je puisse apprendre de nouvelles choses. & & & & & & & \\
\hline PCO & 2. Si j'étudie correctement, je serai capable d'apprendre le contenu de ma formation. & & & & & & & \\
\hline PVT & 3. Je pense que je serai capable d'utiliser mes connaissances acquises lors de cette formation dans d'autres formations. & & & & & & & \\
\hline SEP & 4. Je crois que je vais obtenir une excellente note dans cette formation. & & & & & & & \\
\hline SEP & 5. Je suis convaincu que je peux comprendre les notions les plus difficiles de cette formation. & & & & & & & \\
\hline ME & 6. Obtenir une bonne note ou appréciation dans cette formation est la chose la plus satisfaisante pour moi à l'heure actuelle. & & & & & & & \\
\hline PCO & 7. Je suis responsable si je n'apprends pas le contenu de cette formation. & & & & & & & \\
\hline PVT & 8. C'est important pour moi d'apprendre le contenu de cette formation. & & & & & & & \\
\hline ME & 9. Ma principale préoccupation est d'obtenir une bonne note dans cette formation. & & & & & & & \\
\hline SEP & 10. Je suis sûr que je peux apprendre les notions de base enseignées dans ce cours. & & & & & & & \\
\hline ME & 11. Si je suis capable, j'aimerais avoir les meilleures notes ou appréciations que la plupart des étudiants dans cette formation. & & & & & & & \\
\hline SEP & $\begin{array}{l}\text { 12. J'ai confiance dans le fait de pouvoir apprendre les notions les plus complexes présentées par les enseignants dans cette } \\
\text { formation. }\end{array}$ & & & & & & & \\
\hline MI & 13. Dans une formation comme celle-ci, je préfère que le contenu pique ma curiosité, même s'il est difficile à apprendre. & & & & & & & \\
\hline PVT & 14. Je suis intéressé(e) par le contenu de la formation. & & & & & & & \\
\hline PCO & 15. Si je fais assez d'effort, je vais comprendre le contenu de cette formation. & & & & & & & \\
\hline SEP & 16. J'ai confiance dans le fait que je peux très bien réussir cette formation. & & & & & & & \\
\hline SEP & 17. Je prévois de bien réussir cette formation. & & & & & & & \\
\hline MI & 18. Ce qui est le plus important pour moi est d'essayer de comprendre en profondeur la matière de ce cours. & & & & & & & \\
\hline PVT & 19. Je comprends l'utilité d'apprendre le contenu de cette formation & & & & & & & \\
\hline MI & $\begin{array}{l}\text { 20. Dans cette formation, lorsque j'en ai la possibilité, je choisis les thématiques qui me font le plus apprendre même si je ne suis pas } \\
\text { certain d'avoir une bonne note. }\end{array}$ & & & & & & & \\
\hline $\mathrm{PCO}$ & 21. Si je ne comprends pas le contenu de la formation, c'est parce que je n'ai pas travaillé assez fort. & & & & & & & \\
\hline PVT & 22. J'aime le contenu de cette formation. & & & & & & & \\
\hline PVT & 23. Comprendre le contenu de cette formation est très important pour moi. & & & & & & & \\
\hline SEP & 24. Je suis convaincu que je peux maîtriser les compétences enseignées dans cette formation. & & & & & & & \\
\hline ME & $\begin{array}{l}\text { 25. Je veux bien réussir dans ce cours parce que c'est important pour moi de démontrer mes capacités à ma famille, à mes amis, à } \\
\text { mes enseignants ou à d'autres personnes. }\end{array}$ & & & & & & & \\
\hline SEP & $\begin{array}{l}\text { 26. Si je prends en compte le degré de difficulté de cette formation, les enseignants et mes habiletés, je crois que je vais réussir ce } \\
\text { cours. }\end{array}$ & & & & & & & \\
\hline
\end{tabular}




\section{Références}

1. République française (2015) Arrêté du 13 novembre 2015 fixant la liste des diplômes d'études spécialisées de médecine. JORF $\mathrm{n}^{\mathrm{o}} 0281 \mathrm{du} 4$ décembre 2015. https://www.legifrance.gouv.fr/ affichTexte.do?cidTexte=JORFTEXT000031560595. (Dernier accès le 6 février 2019)

2. Neraal S, Freund Y (2018) Opinions des étudiants du diplôme d'études spécialisées complémentaires de médecine d'urgence au sujet du nouveau diplôme d'études spécialisées de médecine d'urgence. Ann Fr Med Urgence 8:22-6

3. Ryan RM, Deci EL (2000) Intrinsic and extrinsic motivations: classic definitions and new directions. Contemp Educ Psychol 25:54-67

4. Sobral DT (2004) What kind of motivation drives medical students' learning quests? Med Educ 38:950-7

5. Stegers-Jager KM, Cohen-Schotanus J, Themmen APN (2012) Motivation, learning strategies, participation and medical school performance: motivation, learning strategies and participation. Med Educ 46:678-88

6. Vallerand RJ, Fortier MS, Guay F (1997) Self-determination and persistence in a real-life setting toward a motivational model of high school dropout. J Pers Soc Psychol 72:1161-76

7. Kusurkar RA, Ten Cate TJ, van Asperen M, et al (2011) Motivation as an independent and a dependent variable in medical education: a review of the literature. Med Teach 33:242-62

8. Pelaccia T, Delplancq H, Triby E, et al (2008) La motivation en formation : une dimension réhabilitée dans un environnement d'apprentissage en mutation. Pedag Med 9:103-21

9. Pelaccia T, Viau R (2017) Motivation in medical education. Med Teach 39:136-40

10. Pelaccia T, Delplancq H, Triby E, et al (2009) Impact of training periods in the emergency department on the motivation of health care students to learn. Med Educ 43:462-9

11. Holland C (2016) Critical review: medical students' motivation after failure. Adv Health Sci Educ Theory Pract 21:695-710

12. Pintrich PR, Smith DAF, Garcia T, Mckeachie WJ (1993) Reliability and predictive validity of the motivated strategies for learning questionnaire (MSLQ). Educ Psychol Meas 53:801-13
13. Pelaccia T, Tardif J (2016) Comment [mieux] former et évaluer les étudiants en médecine et en sciences de la santé ? De Boeck supérieur, Louvain-la-Neuve

14. Pintrich PR, De Groot EV (1990) Motivational and self-regulated learning components of classroom academic performance. Int $\mathrm{J}$ Sch Educ Psychol 82:33-40

15. Tiberius RG, Sinai J, Flak EA (2002) The role of teacher-learner relationships in medical education. In: Norman GR, Vleuten M, Newble DI (eds) International Handbook of Research in Medical Education. Kluwer, Dordrecht

16. Chamberland M, Hivon R (2005) Les compétences de l'enseignant clinicien et le modèle de rôle en formation clinique. Pedag Med 6:98-111

17. Xu G, Rattner SL, Veloski JJ, et al (1995) A national study of the factors influencing men and women physicians' choices of primary care specialties. Acad Med 70:398-404

18. Campos-Outcalt D, Senf J, Watkins AJ, Bastacky S (1995) The effects of medical school curricula, faculty role models, and biomedical research support on choice of generalist physician careers: a review and quality assessment of the literature. Acad Med 70:611-9

19. Gallagher EJ, Goldfrank LR, Anderson GV, et al (1994) Role of emergency medicine residency programs in determining emergency medicine career choice among medical students. Ann Emerg Med 23:1062-7

20. Svirko E, Lambert T, Brand L, Goldacre MJ (2014) Career choices for emergency medicine: national surveys of graduates of 19932009 from all UK medical schools. Emerg Med J 31:556-61

21. Wright B (2004) Career choice of new medical students at three Canadian universities: family medicine versus specialty medicine. Can Med Assoc J 170:1920-4

22. Choucair J, Nemr E, Sleillaty G, Abboud M (2007) Choix de la spécialité en médecine : quels facteurs influencent la décision des étudiants ? Pedag Med 8:145-55

23. Cerasoli CP, Nicklin JM, Ford MT (2014) Intrinsic motivation and extrinsic incentives jointly predict performance: a 40 -year meta-analysis. Psychol Bull 140:980-1008

24. Bandura A (1997) Self-efficacy: the exercise of control. Worth Publishers, Alberta 